\title{
Review
}

Neuropsychobiology

\section{Advanced Analysis of Pharmaco-Sleep Data in Humans}

\author{
Peter Anderer \\ Department of Psychiatry and Psychotherapy, Medical University of Vienna, Siesta Group Schlafanalyse GmbH, and \\ Sleep and Respiratory Care, Home Healthcare Solutions, Philips Austria GmbH, Vienna, Austria
}

\section{Key Words}

Sleep macrostructure · Sleep microstructure - Sleep

classification · Interrater reliability · Validity

\begin{abstract}
Pharmaco-sleep studies in humans aim at the description of the effects of drugs, most frequently substances that act on the central nervous system, by means of quantitative analysis of biosignals recorded in subjects during sleep. Up to 2007 , the only standard for the classification of sleep macrostructure that found worldwide acceptance were the rules published in 1968 by Rechtschaffen and Kales. In May 2007, the AASM Manual for the Scoring of Sleep and Associated Events was published by the American Academy of Sleep Medicine, and concerning the classification of sleep stages, these new rules are supposed to replace those developed by Rechtschaffen and Kales. As compared to the rather low interrater reliability of manual sleep scoring, semiautomated approaches may achieve a reliability close to 1 (Cohen's kappa 0.99 for 2 semiautomated scorings as compared to 0.76 for 2 manual scorings) without any decline in validity. Depending on the aim of the pharmaco-sleep study, additional analyses concerning sleep fragmentation, sleep microstructure, sleep depth, sleep processes and local aspects of sleep should be considered. For some of these additional features, rules for visual scoring have been established, while for oth-
\end{abstract}

ers automatic analysis is obligatory. Generally, for reasons of cost-effectiveness but also reliability, automatic analysis is preferable to visual analysis. However, the validity of the automatic method applied has to be proven.

(c) 2016 S. Karger AG, Basel

\section{Introduction}

At the behavioral level, sleep can be defined as a 'reversible behavioral state of perceptual disengagement from and unresponsiveness to environmental stimuli' [1]. The sleep-wake cycle and the structure of sleep reflect the spontaneous activity of autoregulatory central nervous system processes. Within sleep, 2 separate states have been defined on the basis of a constellation of physiological parameters. These 2 states, nonrapid eye movement (NREM) and rapid eye movement (REM) sleep, are distinct from one another as each is from wakefulness.

Conventionally, NREM sleep is subdivided into 4 stages - S1, S2, S3 and S4 according to Rechtschaffen and Kales [2] - or 3 stages - N1, N2 and N3 according to the American Academy of Sleep Medicine (AASM) [3]. They reflect light sleep (S1 and N1), middle-deep sleep (S2 and N2) and deep sleep (S3, S4 and N3), respectively. These stages are distinguished from each other principally on the basis of their different patterns of brain electrical ac-

\section{KARGER 125}

C) 2016 S. Karger AG, Base

$0302-282 X / 16 / 0724-0178 \$ 39.50 / 0$
Dr. Peter Anderer

Department of Psychiatry and Psychotherapy University of Vienna

Währinger Gürtel 18-20, AT-1090 Vienna (Austria)

E-Mail peter.anderer@meduniwien.ac.at 
tivity, as measured by electroencephalography (EEG). Indeed, EEG is considered the 'core measurement of polysomnography (PSG)' by Carskadon and Rechtschaffen [4]. The EEG pattern in NREM sleep is synchronous, with characteristic waveforms such as sleep spindles, $\mathrm{K}$ complexes and slow-frequency, high-amplitude waves (delta waves). It is characterized by a periodic alteration of socalled up- and downstates (the 'slow oscillation', which seems to orchestrate the appearance of the aforementioned waveforms) [5-7].

By contrast, REM sleep is defined by EEG activation, muscle atonia and episodic bursts of rapid eye movements. Generally, REM sleep is not subdivided into substates, although tonic and phasic types of REM sleep are often distinguished for research purposes. This distinction between tonic and phasic REM sleep is based on short, transient events occurring in clusters separated by episodes of relative quiescence [1].

In addition to sleep macrostructure described by the sleep stages, sleep microstructure may be described by EEG arousals or by cyclic alternating patterns (CAPs). According to the conceptual framework of the criteria developed by the American Sleep Disorders Association [8], arousals are a marker of sleep disruption, and thus the number of arousals per hour of sleep represents a measure of sleep fragmentation. Also during normal sleep a certain number of arousals - often associated with changes in body position - is found, but excessive numbers of arousals disturb sleep considerably. Arousals are defined as transient shifts in EEG frequencies for a minimum of $3 \mathrm{~s}$, which may be accompanied by an increase in EMG muscle tone. They may occur during any sleep stage. Arousals in REM sleep require a concurrent increase in submental electromyography (EMG). These arousal definitions by the American Sleep Disorders Association have been included in the AASM manual without any changes [3]. According to an alternative conceptual framework suggested by Terzano et al. [9] and Halász et al. [10], arousals embedded in CAP sequences are viewed as elements weaved into the texture of sleep taking part in the regulation of the sleep process. CAPs are defined as sequences of EEG activity in NREM sleep which repeat themselves cyclically. Each cycle of the sequence consists of phase A (transient EEG events constituting a central nervous system arousal) and phase B (background EEG activity). The scoring rules and recording techniques for CAPs have been published as a consensus report [9].

Diagnosis and differential diagnosis of the vast majority of sleep disorders require recording and analyzing additional biosignals. For this so-called cardiorespiratory

Advanced Analysis of Pharmacosleep Data in Humans
PSG, a minimum of 12 physiological signals is needed. Sleep evaluations may comprise additional variables such as electrocardiography, pulse, snoring, airflow, respiratory effort (chest and abdomen), esophageal pressure, oxymetry, body position, (periodic) leg movements, nocturnal penile tumescence, temperature, hormones, electrolytes, etc. These additional signals may be selected on the basis of the disorders to be diagnosed in a particular patient. Further EEG leads are useful for evaluating the spatial distribution of sleep-related EEG patterns. In sleep-related epilepsy or other neurological disorders with associated sleep disturbances, additional EEG leads are indispensable.

Pharmaco-sleep research based on manual and/or computer-assisted evaluation of the sleep EEG has been performed in both healthy volunteers and patients with sleep disorders since the late 1960s $[11,12]$. The objectives of these studies are multifarious such as supporting registration of a new drug, providing evidence of efficacy, evaluating central nervous system side effects or providing basic pharmacodynamic data at an early stage in drug development [13-15]. Generally, pharmacosleep studies have an important role in characterizing the effect of central nervous system-active drugs since an influence on sleep initiation, continuity and architecture may have a significant impact on the clinical use of the drug $[16,17]$.

\section{Recording}

As published by Rechtschaffen and Kales [2] in 1968, at least one central EEG channel (C3-A2 or C4-A1) is obligatory for sleep scoring in humans. In addition, 2 electro-oculography (EOG) leads are required in order to distinguish between eye movements and prefrontal EEG interference. The EOG electrodes are arranged in a way that eyeball movements result in signals going in opposite directions, whereas head movement and EEG artifacts produce signals going in the same direction. One electromyogram lead placed on the chin is required for detection of REM sleep atonia.

Due to the high temporal resolution of the EEG, microstructures of sleep, such as slow waves, $\mathrm{K}$ complexes, sleep spindles, vertex sharp waves or sawtooth waves can be identified and separated from alpha bursts, microarousals, CAP phases or artifacts. To allow these discriminations, the sampling rate of the EEG recording has to be high enough to enable the identification of waveform, amplitude, frequency and duration of the events with ad- 
equate accuracy. A time resolution in the range of milliseconds, for instance, is necessary to discriminate a $\mathrm{K}$ alpha complex (indicating an arousal) from a K complex with a superimposed sleep spindle (indicating consolidated sleep). According to the AASM manual, the minimal sampling rate for EEG, EOG and EMG channels is $200 \mathrm{~Hz}[3]$.

While the value of a high temporal resolution of the electroencephalogram is unequivocally accepted, that of its spatial resolution is still underestimated. According to the AASM manual [3], whose rules are intended to replace those developed by Rechtschaffen and Kales, only 2 additional EEG channels, one frontal lead (F4-A1) and one occipital lead (O2-A1), are required for scoring sleep. The occipital derivation should be used for identifying alpha activity and the frontal derivation for identifying $\mathrm{K}$ complexes and slow-wave activity.

The addition of a parietal EEG electrode makes it possible to evaluate brain topography along the anterior-posterior axis by re-referencing the EEG leads to bipolar derivations (e.g. F3-C3, C3-P3, P3-O1). Such fronto-occipital EEG power gradients in the sleep of young healthy subjects demonstrated topographic power shifts at NREM-REM sleep transitions as well as across and within NREM periods [18]. Since the regional EEG power spectra showed state-related and frequency-specific differences, the study highlighted the additional information that may be gained by a - though rather limited spatial analysis of sleep EEG data. As early as in 1993, a topographic study performed by our group with $18 \mathrm{EEG}$ leads showed an increase in delta power (which was most pronounced frontally) and a decrease in alpha power (which was most pronounced parieto-occipitally) from sleep stages S1 to S4 [19]. A follow-up study demonstrated that not only slow waves, but also sleep spindles were not uniformly distributed across the scalp. Slow sleep spindles were generally distributed over anterior and fast sleep spindles over parietal regions [20]. By means of lowresolution brain electromagnetic tomography developed by Pascual-Marqui et al. [21], we identified cortical spindle sources predominantly medially in the frontal and parietal lobes. Weaker bilateral frontal and parietal sources showed a left-hemispheric predominance [22]. Interestingly, the prefrontal sources (Brodmann areas 9 and 10) oscillated with a frequency below $13 \mathrm{~Hz}$, and the precuneus sources (Brodmann area 7), with a frequency above $13 \mathrm{~Hz}$. These topographic and tomographic studies indicate that the neuronal processes underlying the sleep EEG differ between brain regions, which supports the hypothesis of local aspects of sleep.
Consequently, a comprehensive sleep analysis should include spatial information. Today, a number of sleep recording systems allow the acquisition of data from multiple EEG channels, and thus, limiting factors for increasing the number of electrodes are subjects' comfort, the time needed for applying the electrodes and the increasing time and effort required for artifact handling. In 2004, Huber et al. [23] published a study using a 256-channel EEG recording system with an electrode cap for revealing local changes in slow-wave activity in a night after a learning task of rotation adaptation. The high-density EEG analysis revealed a remarkably stable slow-wave activity that was most pronounced over frontal regions, confirming our early topographic delta distribution obtained with 18 electrodes. Indeed, caps and electrodes with improved comfort and systems combining PSG and highdensity EEG devices are now available and will hopefully stimulate new studies on the spatial analysis of sleep EEG.

Recommendations, specifically intended for pharmaco-sleep studies in man, for subject selection, environmental conditions and recording settings including adaptation nights as well as for digital recordings, calibration and biosignals, can be found in the 'Guidelines for the recording and evaluation of pharmaco-sleep studies in man' recently published by the International PharmacoEEG Society [24].

\section{Analysis}

In any pharmaco-sleep study, the first step of the analysis has to be the classification of sleep stages. Up to 2007, the only standard for the classification of sleep macrostructure that found worldwide acceptance were the rules published by Rechtschaffen and Kales [2] entitled A Manual of Standardized Terminology, Techniques and Scoring System for Sleep Stages of Human Subjects in 1968. In May 2007, the AASM Manual for the Scoring of Sleep and Associated Events was published by the AASM [3]. Concerning the visual classification of sleep stages, these new rules are intended to replace those developed by Rechtschaffen and Kales. However, there are still ongoing discussions on the pros and cons of the visual AASM rules, and thus, no final recommendation for using the Rechtschaffen and Kales or the AASM rules can be given at present. For further commentaries on the AASM manual for scoring sleep, see those by Schulz [25] and Parrino et al. [26]. The effects of the scoring rules on interrater reliability were evaluated by Danker-Hopfe et al. [27]. The authors summarize that on the one hand the integration of frontal, 
central and occipital leads improves interrater reliability. On the other hand, however, this advantage is counteracted by the impairment of interrater reliability due to the new rule according to which any cortical arousal, whether or not it is associated with an EMG increase, determines the end of stage N2. Cohen's kappa was 0.76 for visual scoring according to the AASM standard as compared with 0.68 for visual scoring according to Rechtschaffen and Kales. To allow a direct comparison, both kappa values were based on 5 classes, i.e. for Rechtschaffen and Kales scorings stage S3 and S4 were combined with a slowwave sleep stage, and epochs scored as movement time were ignored. In a second paper, Moser et al. [28] investigated the effects of the scoring standard on sleep parameters in the same data set. In summary, the new standard showed only a minor influence on total sleep time, sleep efficiency and stage of REM sleep, but affected wake after sleep onset as well as the distribution of NREM sleep stages (stage 1 and slow-wave sleep increased by approx. 10 min each, stage 2 sleep decreased by approx. $20 \mathrm{~min}$ ). In the process of adapting the automated sleep staging system Somnolyzer $24 \times 7$ - which in 2005 had been developed and validated for scoring sleep according to Rechtschaffen and Kales - to the new AASM rules, we performed a stage transition analysis to evaluate step by step the effects the change of the rules had on sleep staging $[29,30]$. On average, the inclusion of the occipital lead for detecting alpha activity affected only $2-3$ epochs per recording. In these rare cases, epochs changed from stage $\mathrm{S} 1$ to stage $\mathrm{W}$, since the occipital alpha activity was not detectable at central leads. In the vast majority of cases, however, alpha activity was detectable not only at occipital, but also at central leads and thus the inclusion of the occipital lead had only a minor effect on sleep stage scoring. More epochs were affected by the addition of the frontal lead, as epochs with slow waves (just) below $75 \mu \mathrm{V}$ at central leads may change from S2 to N3 if their amplitudes are above $75 \mu \mathrm{V}$ at frontal leads. As a consequence, an average of 19 epochs per recording changed from S2 to N3. The exact influence of the change in the scoring rules depends on individual EEG characteristics, such as the anterior-posterior gradient of slow-wave amplitudes or densities of sleep spindles and $\mathrm{K}$ complexes on the one hand, and arousal density and alpha topography on the other, and may range from nearly no changes at all to increases in slow-wave sleep of up to $53 \mathrm{~min}$ and in stage 1 sleep of up to $44 \mathrm{~min}$, with a maximal decrease in stage 2 sleep of up to $55 \mathrm{~min}$ [28-30]. In any case, scoring results obtained with one method cannot directly be compared with those obtained by the other.

Advanced Analysis of Pharmacosleep Data in Humans
Since the first publication of the Rechtschaffen and Kales scoring standards in 1968, numerous attempts at a computer-assisted identification of sleep stages have been published. According to Schulz [25], sleep experts should concentrate on 3 major tasks related to automated sleep scoring: (1) definition and development of an algorithm, (2) surveillance of the analyzing process, including artifact decontamination, and (3) quality control of sleep analysis. In the paper on digital analysis and technical specifications, which accompanied the AASM manual of 2007, Penzel et al. [31] presented a comprehensive review of papers on computer application and validation of sleep staging. The authors identified 119 papers suitable for evidence review and defined a grading for performance evaluation (from level 1 with a sample size $>64$ without nesting within subject; sequential or representative sample; event-by-event or epoch-by-epoch comparison to level 5 for case series) with 8 additional evaluation factors for grading within level: (1) normal controls and clinically relevant group used in sample; (2) no recording selection for quality and/or discarded $<5 \%$ of records; (3) clinical standard used for group classification; (4) standard used for sleep scoring and recording; (5) blind, independent scoring; (6) multiple human scorers used to set comparison standard; (7) entire recordings used, and (8) description adequate for replication. The paper on the automated sleep classification system Somnolyzer $24 \times 7$ was assigned grading level 1 and listed on top of 44 papers on general computer applications for sleep staging [31]. Indeed, the paper published by Anderer et al. [29] was the only study that fulfilled all criteria for level 1 and all 8 additional evaluation factors for grading within level 1. By then, also the study published in 2010 reporting the validation of the AASM version of the Somnolyzer system met all these criteria [30].

Since the automated classification by Somnolyzer typically requires only few corrections during visual editing, interrater reliability of 2 Somnolyzer-assisted sleep scorings is close to 1 . To perform the standardized quality control procedure most efficiently, sleep experts receive information from various steps of the analysis including raw data, artifacts and sleep/wake-related features (see fig. 1 for an example). While on average between 1 and $5 \%$ of the epochs are changed during expert review, in single cases up to $20 \%$ of the epochs might be affected, and thus, at present, unsupervised automatic analysis is not recommended. Cohen's kappa for interrater reliability between 2 Somnolyzer-assisted classifications was 0.99 for Rechtschaffen and Kales scorings based on 286 PSGs [29] and again 0.99 for AASM scorings based on 72

Neuropsychobiology 2015;72:178-187 181 


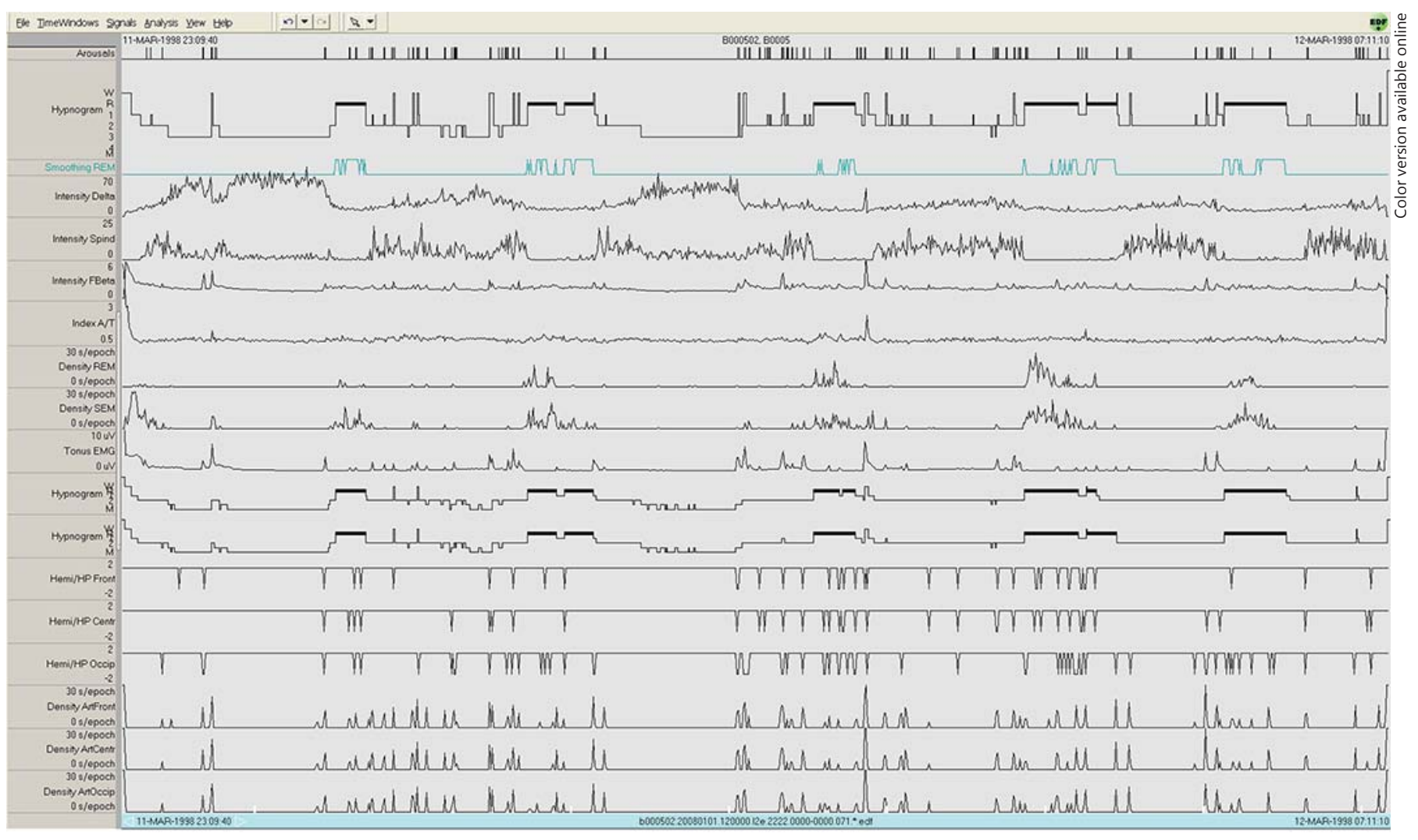

Fig. 1. The Somnolyzer $24 \times 7$ traces for the expert review procedure (22-year-old male subject). In addition to arousals and the hypnogram according to the AASM manual [45], various features are displayed for the entire night ( $8 \mathrm{~h} 1 \mathrm{~min} 30 \mathrm{~s}$ ): smoothing REM (the marked epochs were classified as REM due to the rules for the start and end of a REM period); intensity of delta waves $(0.5-2 \mathrm{~Hz}$, Intensity Delta); intensity of spindle episodes (Intensity Spind); intensity of fast beta waves $(20-40 \mathrm{~Hz}$, Intensity FBeta); quotient of alpha/theta intensity (Index A/T); density of REMs; density of slow eye movements (SEMs); trimmed group mean of tonic chin EMG activity (Tonus EMG); an intermediate hypnogram based on EEG, EOG and EMG channels; a second intermediate hypnogram based on EEG and EOG channels only; indications whether the EEG channel of the default (plus values) or the alternative (minus values) hemisphere has been used for classification. If the values were \pm 2 instead of \pm 1 , an additional high-pass (HP) filter was applied to reduce sweat artifacts (Hemi/HP Front, Hemi/HP Centr and Hemi/HP Occip for the frontal, central and occipital EEG channels, respectively); density of detected artifacts in the frontal, central and occipital channels (Density ArtFront, Density Art Centr, Density ArtOccip). As a first step of the expert review, the plausibility of the staging is evaluated by means of these traces. Note for instance the high alpha/theta index at the beginning of the recording with a steep decline towards sleep onset. On the other hand, the SEM density is low at lights out and increases rapidly to its maximal value at sleep onset. Interestingly, even after short awakenings, increases in SEM density are observed. Moreover, SEM as well as REM density increases during REM periods. While delta intensity is maximal in NREM sleep N3, sleep spindle density is maximal in NREM sleep N2. Due to the rather few epochs with artifacts, the Somnolyzer switched only in rare cases from the EEG channels in the default (right) hemisphere to the EEG channel in the alternative (left) hemisphere. For further details, see Anderer et al. $[29,30]$.
PSGs [30]. In contrast, interrater reliability for 2 manual scorings resulted in Cohen's kappa of 0.68 for the 286 Rechtschaffen and Kales studies and 0.76 for the 72 AASM studies [29, 30]. Cohen's kappa for the agreement between the 2 Somnolyzer-assisted and the 2 visual scorings for the 72 AASM studies was between 0.75 and 0.76 and thus was comparable to the interrater reliability between 2 manual scorings. Thus, the validity of Somnolyzer-as- sisted scoring is comparable to that of human experts, while the reliability is close to 1 . Since the time required for expert review of the automated Somnolyzer scoring is at least 5 times shorter than for manual scoring, Somnolyzer-assisted scoring reduces interrater variability as well as scoring time to a minimum. In pharmaco-sleep studies, typically comparing drug-induced versus placebo-induced changes, the reliability of PSG scoring is essential. 


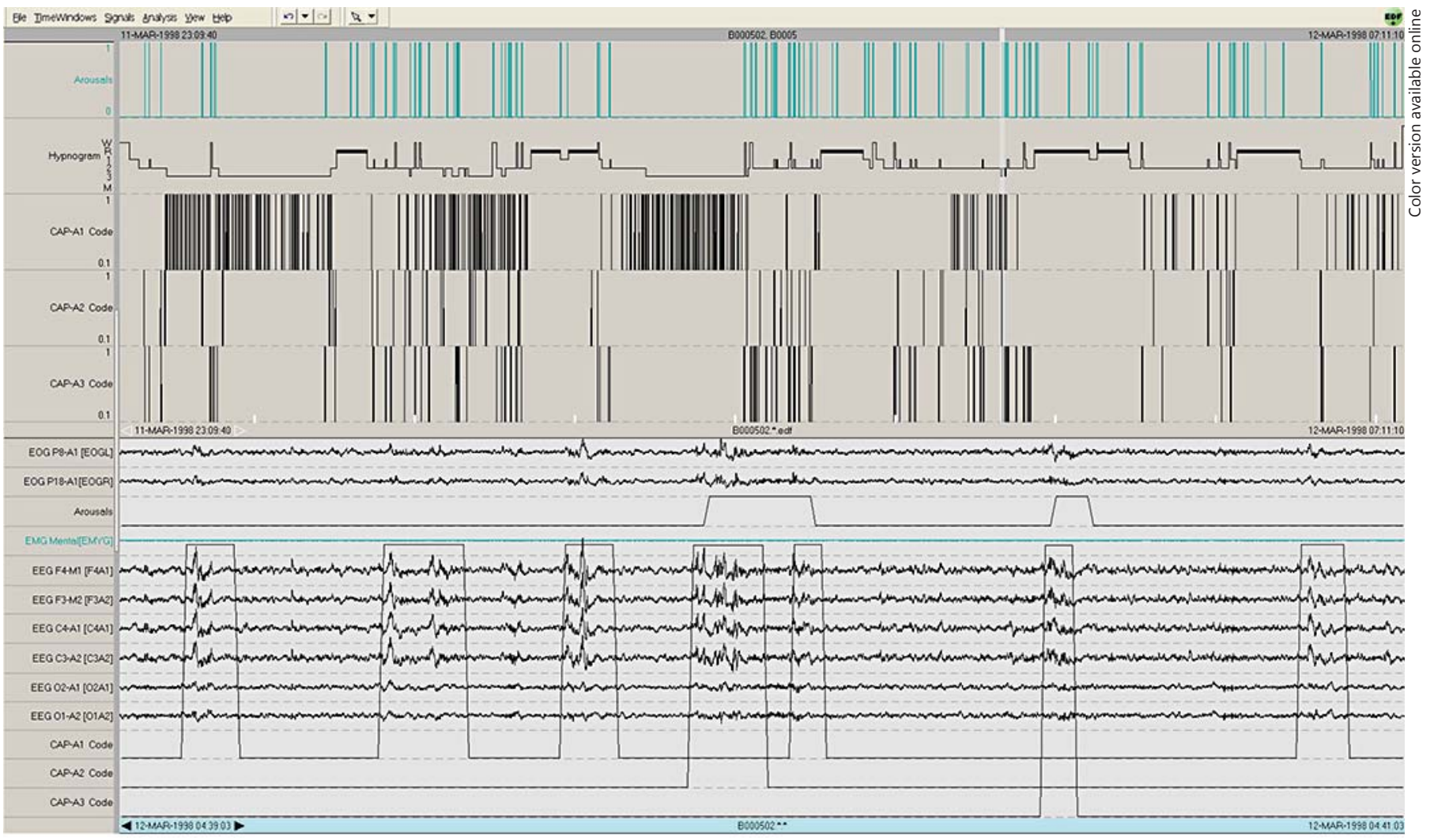

Fig. 2. Arousals and CAPs in a period of consolidated NREM sleep (22-year-old male subject). In the upper window, in addition to the arousals and the hypnogram according to the AASM manual [3], CAP A1, A2 and A3 phases according to Terzano et al. [9] are displayed for the entire night ( $8 \mathrm{~h} 1 \mathrm{~min} 30 \mathrm{~s})$. In the lower window, raw data (left and right EOG, chin EMG, F4-A1, F3-A2, C4-A1, C3-A2, O2-A1, O1-A2 EEG) as well as the automatically detected arousals and CAP A phases are displayed for a 2-min window. Note that the 5 identified CAPs of subtype A1 include delta bursts and $\mathrm{K}$ complex sequences with $<20 \%$ of EEG desynchrony, the CAP A2 shows more than $20 \%$ but less than $50 \%$ of EEG desynchrony and the CAP A3 shows EEG desynchrony for more than $50 \%$ of the phase A duration.
First applications of Somnolyzer in a double-blind, placebo-controlled pharmacosleep study on the potential interaction of $20 \mathrm{mg}$ paroxetine and $1 \mathrm{mg}$ alprazolam in healthy young volunteers and in a single-blind, placebocontrolled clinical sleep study on the acute effects of 100 $\mathrm{mg}$ trazodone in patients with nonorganic insomnia related to somatoform pain disorder confirmed the validity of the classifier for scoring PSGs after treatment with psychotropic drugs $[32,33]$. This validity was further confirmed by Svetnik et al. [34] in 164 PSGs of 82 subjects in a clinical trial using zolpidem in a phase advance model of transient insomnia. Recently, Somnolyzer-assisted scoring has been successfully used in a multicenter, double-blind, randomized, placebo-controlled, 2-way crossover study in 161 primary insomnia patients with the dual orexin receptor antagonist almorexant [35].

Advanced Analysis of Pharmacosleep Data in Humans
Irrespective of the method used for analyzing sleep EEG data, the significance of the results critically depends on the treatment of artifacts. It is obvious that analysis of data contaminated by artifacts can lead to spurious results. In sleep EEG data, simple artifact elimination methods based on the definition of a threshold for maximal EEG amplitudes, as frequently used in evoked potential studies for instance, are certainly not appropriate. As part of the EU-funded project SIESTA, we reviewed in detail types and treatment of artifacts in the sleep EEG [36]. A reliable and valid artifact processing strategy should include: (1) high-quality recording techniques in order to minimize the occurrence of avoidable artifacts (e.g. technical artifacts); (2) artifact minimization procedures in order to minimize the loss of data by estimating the interference of different artifacts in the EEG recordings, thus 


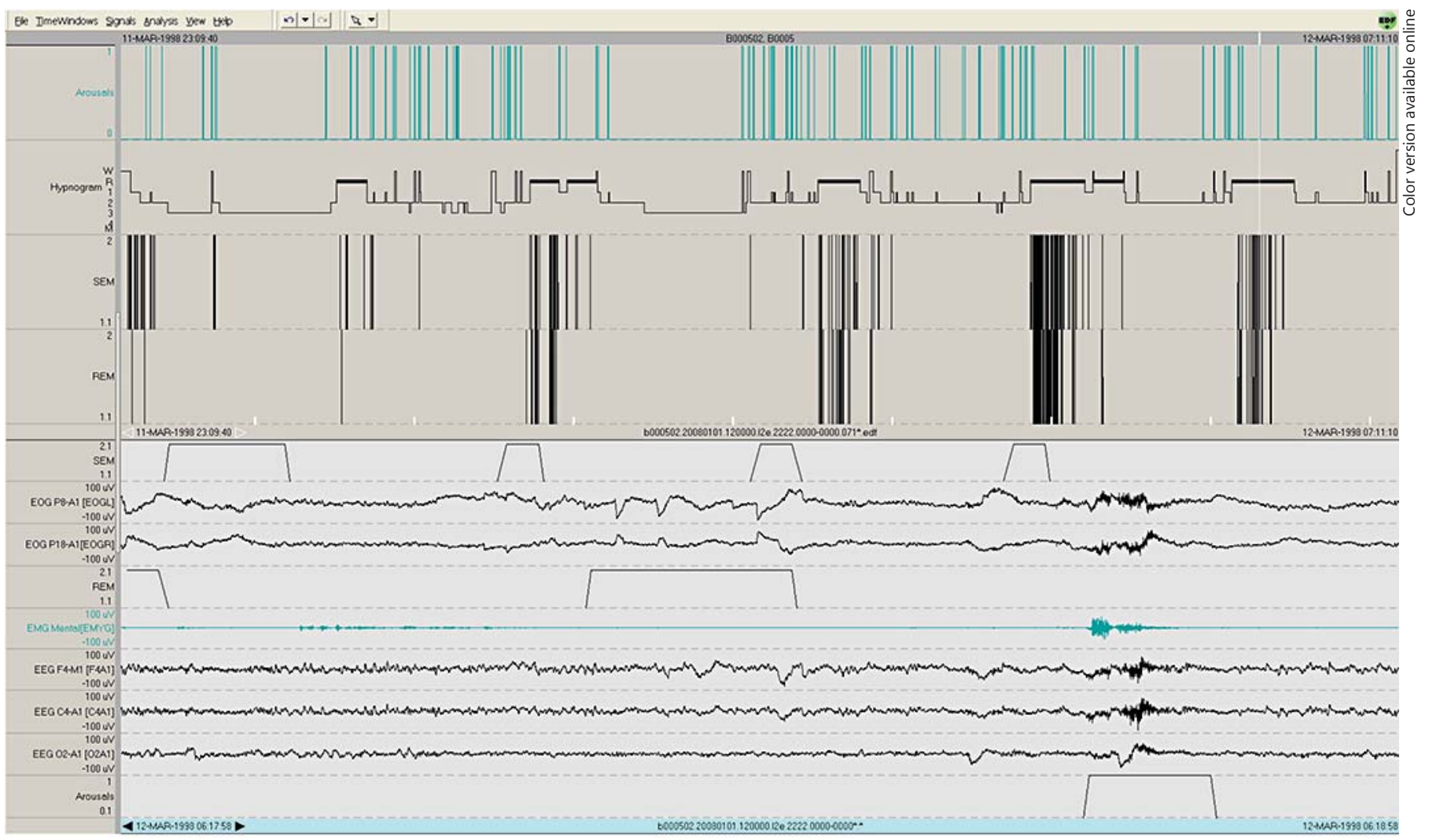

Fig. 3. REMs and SEMS in a period of phasic REM sleep (22-yearold male subject). In the upper window, in addition to the arousals and the hypnogram according to the AASM manual [45], automatically detected SEM and REM events are displayed for the entire night ( $8 \mathrm{~h} 1 \mathrm{~min} 30 \mathrm{~s}$ ). In the lower window, raw data (left and right EOG, chin EMG, F4-A1, C4-A1, O2-A1 EEG) as well as the automatically detected arousals and SEM and REM events are dis- played for a 1-min window. Note that in REM sleep, arousals have to be associated with an increase in chin EMG activity. The Somnolyzer defines REMs and SEMs with a temporal resolution of $2 \mathrm{~s}$. Note that REMs might be superimposed on SEMs. Thus, as can be seen in the upper part, SEMs do not only occur at sleep onset or after awakenings, but are also characteristic features of REM sleep (compare also Pizza et al. [46]). allowing the calculation of the 'corrected' EEG (e.g. ocular and electrocardiographic artifacts), and finally (3) artifact identification procedures in order to define and eliminate epochs contaminated by remaining artifacts (e.g. movement and muscle artifacts). Indeed, artifacts can mimic almost any kind of EEG pattern $[37,38]$ and artifacts included in automatic analysis can seriously affect the results. For instance, Brunner et al. [39] demonstrated that the rejection of short-lasting muscle bursts significantly reduced power spectral density in all frequencies from 0.25 to $32 \mathrm{~Hz}$, most prominently of course in the faster frequency bands. Thus, the careful handling of artifacts is of the utmost importance for EEG data processing.

For a review on automated detection and analysis of EEG waveforms such as arousals, CAPs, sleep spindles, $\mathrm{K}$ complexes, slow waves, as well as spike and wave activity or specific pattern related to hypoglycemia, see Penzel et al. [31]. In our paper in the textbook for the training course of the International Pharmaco-EEG Society in 2006, we presented methods and validation data for the automatic detection of a variety of patterns such as the artifact detector, the spindle detector, the K complex detector, the detector for rapid and slow eye movements (REMs and SEMs) as well as the arousal detector [40]. In addition to the hypnogram according to the AASM standard, figure 2 displays arousals and CAP A phases for a stable NREM period, figure 3 REMs and SEMs for a phasic REM period.

Last but not least, computer applications of the sleep EEG may provide insight into aspects of the signal that are not recognizable by visual inspection of the EEG, for 


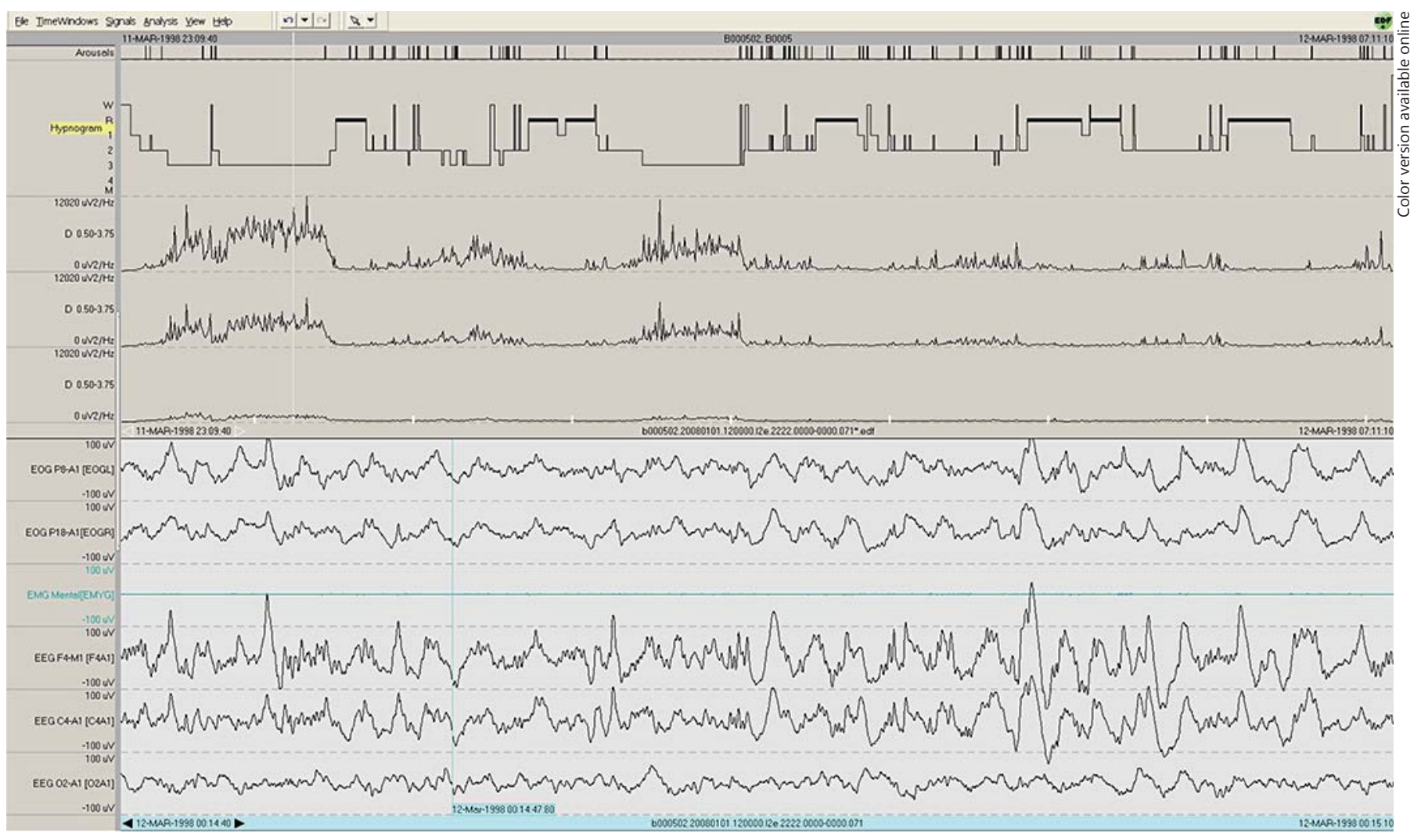

Fig. 4. Delta power at frontal, central and occipital leads (22-yearold male subject). In the upper window, in addition to the arousals and the hypnogram according to the AASM manual [45], delta power $(0.5-3.5 \mathrm{~Hz})$ for F4-A1 (upper trace), C4-A1 (middle trace) and $\mathrm{O} 2-\mathrm{A} 1$ (lower trace) are displayed for the entire night $(8 \mathrm{~h}$ $1 \mathrm{~min} 30 \mathrm{~s}$ ). In the lower window, raw data (left and right EOG, chin EMG, F4-A1, C4-A1, O2-A1 EEG) are displayed for a 30-second window. Note that the maximal delta power is seen at the frontal lead and in the first sleep cycle. Indeed, delta power is maximal over frontal brain regions (compare Zeitlhofer et al. [19] and Huber et al. [23]) and is modulated by the sleep cycles with a declining trend in the course of the night (see also Achermann [41]). example the description of sleep with a better time resolution than $30 \mathrm{~s}$ using continuous sleep analyzers based on complexity measures, autoregressive models, hidden Markov models or gaussian mixture models [40]. However, the sleep electroencephalogram is most frequently quantified by means of spectral analysis, which decomposes it into its constituent frequency components (see Achermann [41] for theory and application of spectral analysis on sleep EEG). Typically, fast Fourier transform is applied to short EEG segments (usually between 2 and $10 \mathrm{~s}$ ), and the power spectral density spectrum (scaled in $\mu \mathrm{V}^{2} / \mathrm{Hz}$ ) is averaged over artifact-free segments for a given time (e.g. for a 30-second epoch) or condition (e.g. for NREM or REM periods). Based on the averaged spectra, target variables such as absolute power (in $\left.\mu \mathrm{V}^{2}\right)$ and relative power (in \% of total power) may be derived for various frequency bands and displayed as a function of time for an individual EEG channel (see fig. 4 for the distribution of delta power in the course of the night). In recent years, studies on REM sleep behavior disorder showed increased evidence that the loss of REM sleep atonia may be an early manifestation of degenerative neurological disease $[42,43]$. A simple measure to automatically quantify the time in REM sleep without atonia - the atonia index - was developed and validated by Ferri et al. [44] in 2010 .

\section{Conclusion}

Technical and digital specifications, including sensor types, maximal electrode impedance, minimum digital resolution, sampling rates and filter settings for routine PSG recordings were summarized in the 2007 AASM 
Manual for the Scoring of Sleep and Associated Events and its update in 2012 [3, 45]. Specific recommendations for pharmaco-PSG studies can be found in the 'Guidelines for the recording and evaluation of pharmaco-sleep studies in man: the International Pharmaco-EEG Society' [24].

In pharmaco-sleep studies, sleep classification should be performed at specialized centers by experienced, trained and certified scorers. While unsupervised automatic scoring cannot be recommended for sleep staging at present, automatic sleep analysis has proven its usefulness in a semiautomated scoring approach or for obtaining a second independent scoring. In the first case, visual editing of the automatic scoring has to be performed by experienced and trained scorers, and in the second case, comparisons between visual and automatic scoring can be used to identify problematic recordings that should be considered for consensus scoring [30]. Obviously, an automatic method that requires only few corrections during visual editing should be used so that the influence of interindividual differences in sleep scoring can be reduced to a minimum. Specifically in pharmaco-sleep studies, both the validity and the reliability of sleep scoring are essential.
The main hypotheses in pharmaco-sleep studies are usually based on measures of sleep derived from Rechtschaffen and Kales or the AASM (e.g. sleep efficiency, wake after sleep onset, percentage of slow-wave sleep, etc.). Nevertheless, additional analyses concerning sleep fragmentation (e.g. arousals, CAPs, etc.), sleep microstructure (e.g. spindles, phasic REM episodes, etc.), sleep depth (e.g. delta plots via fast Fourier transform analysis, 'microcontinuity' of slow waves, probability of slow-wave sleep, etc.), sleep processes (e.g. continuous physiological and probabilistic models of sleep, etc.), REM sleep atonia and local aspects of sleep (e.g. topography, source localization by means of low-resolution brain electromagnetic tomography, etc.) should be considered. For some of these additional features, rules for visual scoring have been established, while for others automatic analysis is obligatory. Generally, for reasons of cost-effectiveness but also reliability, automatic analysis is preferable to visual analysis. However, the validity of the automatic method applied has to be proven. Last but not least, it is important to note that careful handling of artifacts is a prerequisite for all automatic methods [36].

\section{References}

1 Carskadon MA, Dement WC: Normal human sleep: an overview; in Kryger MH, Roth T, Dement WC (eds): Principles and Practice of Sleep Medicine, ed 3. Philadelphia, Saunders Company, 2000, pp 15-26.

2 Rechtschaffen A, Kales A: A Manual of Standardized Terminology, Techniques and Scoring System for Sleep Stages of Human Subjects. Washington, Public Health Service, US Goverment Priting Office, 1968.

3 Iber C, Ancoli-Israel S, Chesson A, Quan SF; American Academy of Sleep Medicine: The AASM Manual for the Scoring of Sleep and Associated Events: Rules, Terminology and Technical Specifications, ed 1. Westchester, American Academy of Sleep Medicine, 2007.

4 Carskadon MA, Rechtschaffen A: Monitoring and staging human sleep; in Kryger MH, Roth T, Dement WC (eds): Principles and Practice of Sleep Medicine, ed 3. Philadelphia, Saunders Company, 2000, pp 1197-1217.

5 Achermann P, Borbély AA: Low-frequency $(<1 \mathrm{~Hz})$ oscillations in the human sleep electroencephalogram. Neuroscience 1997;81: 213-222.

6 Steriade M: Grouping of brain rhythms in corticothalamic systems. Neuroscience 2006; 137:1087-1106.
7 Halász P, Bódizs R, Parrino L, Terzano M: Two features of sleep slow waves: homeostatic and reactive aspects - from long term to instant sleep homeostasis. Sleep Med 2014;15: 1184-1195.

8 American Sleep Disorder Association: EEG arousals: scoring rules and examples. Sleep 1992;15:173-184.

-9 Terzano MG, Parrino L, Sherieri A, Chervin R, Chokroverty S, Guilleminault C, Hirshkovitz M, Mahowald M, Moldofsky H, Rosa A, Thomas R, Walters A: Atlas, rules, and recording techniques for the scoring of cyclic alternating pattern (CAP) in human sleep. Sleep Med 2002;3:187-199.

10 Halász P, Terzano M, Parrion L, Bódizs R: The nature of arousal in sleep. J Sleep Res 2004; 13:1-23.

$\checkmark 11$ Oswald I, Jones HS, Mannerheim JE: Effects of two slimming drugs on sleep. Br Med J 1968;1:796-799.

12 Itil TM, Saletu B, Marasa J: Digital computer analyzed sleep electroencephalogram (sleep print) in predicting anxiolytic properties of clorazepate dipotassium (Tranxene). Curr Ther Res 1972;14:415-427.

13 Rijnbeek B, de Visser SJ, Franson KL, Cohen $\mathrm{AF}$, van Gerven JM: REM sleep effects as a biomarker for the effects of antidepressants in healthy volunteers. J Psychopharmacol 2003; 17:196-203.
14 DeMartinis NA, Winokur A: Effects of psychiatric medications on sleep and sleep disorders. CNS Neurol Disord Drug Targets 2007; 6:17-29.

15 Leiser SC, Dunlop J, Bowlby MR, Devilbiss DM: Aligning strategies for using EEG as a surrogate biomarker: a review of preclinical and clinical research. Biochem Pharmacol 2011;81:1408-1421.

16 Saletu B, Anderer P, Brandstätter N, Frey R, Grünberger J, Klösch G, Mandl M, Wetter T, Zeitlhofer J: Insomnia in generalized anxiety disorder: polysomnographic, psychometric and clinical investigations before, during and after therapy with a long- versus a short-halflife benzodiazepine (quazepam versus triazolam). Neuropsychobiology 1994;29:69-90.

-17 Saletu B, Saletu-Zyhlarz G, Anderer P, Brandstätter N, Frey R, Gruber G, Klösch G, Mandl M, Grünberger J, Linzmayer L: Nonorganic insomnia in generalized anxiety disorder. 2. Comparative studies on sleep, awakening, daytime vigilance and anxiety under lorazepam plus diphenhydramine $\left(\right.$ Somnium ${ }^{\circledR}$ ) versus lorazepam alone, utilizing clinical, polysomnographic and EEG mapping methods. Neuropsychobiology 1997;36:130-152. 
18 Werth E, Achermann P, Borbély AA: Frontooccipital EEG power gradients in human sleep. J Sleep Res 1997;6:102-112.

- 19 Zeitlhofer J, Anderer P, Obergottsberger S, Schimicek P, Lurger S, Marschnigg E, Saletu B, Deecke L: Topographic mapping of EEG during sleep. Brain Topogr 1993;6:123-129.

20 Zeitlhofer J, Gruber G, Anderer P, Asenbaum S, Schimicek P, Saletu B: Topographic distribution of sleep spindles in young healthy subjects. J Sleep Res 1997;6:149-155.

21 Pascual-Marqui RD, Lehmann D, Koenig T, Kochi K, Merlo MCG, Hell D, Koukkou M: Low resolution brain electromagnetic tomography (LORETA) functional imaging in acute, neuroleptic-naïve, first-episode, productive schizophrenia. Psychiatry Res Neuroimag 1999;90:169-179.

-22 Anderer P, Klösch G, Gruber G, Trenker E, Pascual-Marqui RD, Zeitlhofer J, Barbanoj MJ, Rappelsberger P, Saletu B: Low-resolution brain electromagnetic tomography revealed simultaneously active frontal and parietal sleep spindle sources in the human cortex. Neuroscience 2001;103:581-592.

-23 Huber R, Ghilardi MF, Massimini M, Tononi G: Local sleep and learning. Nature 2004;430: 78-81.

24 Jobert M, Wilson FJ, Roth T, Ruigt GSF, Anderer P, Drinkenburg WHIM: Guidelines for the recording and evaluation of pharmacosleep studies in man: the International Pharmaco-EEG Society (IPEG). Neuropsychobiology 2013;67:127-167.

25 Schulz H: Rethinking sleep analysis: comment on the AASM manual for the scoring of sleep and associated events. J Clin Sleep Med 2008;4:99-103.

-26 Parrino L, Ferri R, Zucconi M, Fanfulla F: Commentary from the Italian Association of Sleep Medicine on the AASM manual for the scoring of sleep and associated events: for debate and discussion. Sleep Med 2009;10:799-808.

-27 Danker-Hopfe H, Anderer P, Zeitlhofer J, Boeck M, Dorn H, Gruber G, Heller E, Loretz E, Moser D, Parapatics S, Saletu B, Schmidt A, Dorffner G: Interrater reliability for sleep scoring according to the Rechtschaffen \& Kales and the new AASM standard. J Sleep Res 2009;18:74-84.

-28 Moser D, Anderer P, Gruber G, Parapatics S, Loretz E, Boeck M, Kloesch G, Heller E, Schmidt A, Danker-Hopfe H, Saletu B, Zeitlhofer J, Dorffner G: Sleep classification according to AASM and Rechtschaffen \& Kales: effects on sleep scoring parameters. Sleep 2009;32:139-149.
29 Anderer P, Gruber G, Parapatics S, Woertz M, Miazhynskaia T, Klösch G, Zeitlhofer J, Barbanoj M, Danker-Hopfe H, Himanen SL, Kemp B, Penzel T, Röschke J, Grözinger M, Kunz D, Rappelsberger P, Saletu B, Dorffner G: An E-health solution for automatic sleep classification according to Rechtschaffen and Kales: validation study of the Somnolyzer $24 \times 7$ utilizing the Siesta database. Neuropsychobiology 2005;51:115-133.

30 Anderer P, Moreau A, Woertz M, Ross M, Gruber G, Parapatics S, Loretz E, Heller E, Schmidt A, Boeck M, Moser D, Kloesch G, Saletu B, Saletu-Zyhlarz GM, Danker-Hopfe H, Zeitlhofer J, Dorffner G: Computer-assisted sleep classification according to the standard of the American Academy of Sleep Medicine: validation study of the AASM version of the Somnolyzer $24 \times 7$. Neuropsychobiology 2010;62:250-264.

-31 Penzel T, Hirshkowitz M, Harsh J, Chervin RD, Bufkov N, Kryger M, Malow B, Vittello M, Silber MH, Kushida CA, Chesson AL: Digital analysis and technical specifications. J Clin Sleep Med 2007;3:109-120.

32 Barbanoj MJ, Clos S, Romero S, Morte A, Giménez S, Lorenzo JL, Luque A, Dal-Ré R: Sleep laboratory study on single and repeated dose effects of paroxetine, alprazolam and their combination in healthy young volunteers. Neuropsychobiology 2005;51:134-147.

33 Saletu B, Prause W, Anderer P, Mandl M, Freidl M, Weiss M, Aigner M, Mikova O, Saletu-Zyhlarz GM: Insomnia in somatoform pain disorder: sleep laboratory studies on differences to controls and acute effects of trazodone, evaluated by the Somnolyzer $24 \times 7$ and the Siesta database. Neuropsychobiology 2005;51:148-163.

-34 Svetnik V, Ma J, Soper K, Doran S, Renger JJ, Deacon S, Koblan KS: Evaluation of automated and semi-automated scoring of polysomnographic recordings from a clinical trial using zolpidem in the treatment of insomnia. Sleep 2007;20:1562-1574.

35 Hoever P, Dorffner G, Benes H, Penzel T, Danker-Hopfe H, Barbanoj MJ, Pillar G, Saletu B, Polo O, Kunz D, Zeitlhofer J, Berg S, Partinen M, Bassetti CL, Hogl B, Ebrahim IO, Holsboer-Trachsler E, Bengtsson H, Peker Y, Hemmeter U-M, Chiossi E, Hajak G, Dingemanse J: Orexin receptor antagonism, a new sleep-enabling paradigm: a proof-of-concept clinical trial. Clin Pharmacol Ther 2012;91: 975-985.
6 Anderer P, Roberts SJ, Schlögl A, Gruber G, Klösch G, Herrmann WM, Rappelsberger P, Filz O, Barbanoj MJ, Dorffner G, Saletu B: Artifact processing in computerized analysis of sleep EEG - a review. Neuropsychobiology 1999;3:150-157.

37 Barlow JS: Automatic elimination of electrode-pop artifacts in EEGs. IEEE Trans Biomed Eng 1986;33:517-521.

38 Klass DW: The continuing challenge of artifacts in the EEG. Am J EEG Technol 1995;35: 239-269.

39 Brunner DP, Vasko RC, Detka CS, Monahan JP, Reynolds CF, Kupfer DJ: Muscle artifacts in the sleep EEG: automated detection and effect on all-night EEG power spectra. J Sleep Res 1996;5:155-164.

40 Anderer P, Saletu B, Saletu-Zyhlarz GM, Gruber G, Parapatics S, Miazhynskaia T, Woertz M, Rosipal R, Klösch G, Zeitlhofer J, Dorffner G: Electrophysiological evaluation of sleep; in Kinoshita T (ed): Textbook for the Training Course of the International Pharmaco-EEG Society, September 7\&8, 2006, in the Awaji Island of Hyogo Prefecture, Japan, 2006, pp 107-129.

41 Achermann P: EEG analysis applied to sleep. Epileptologie 2009;26:28-33.

42 McCarter SJ, St Louis EK, Boeve BF: REM sleep behaviour disorder and REM sleep without atonia as an early manifestation of degenerative neurological disease. Curr Neurol Neurosci Rep 2012;12:182-192.

43 Khalil A, Wright MA, Walker MC, Eriksson $\mathrm{SH}$ : Loss of rapid eye movement sleep atonia in patients with REM sleep behavioural disorder, narcolepsy, and isolated loss of atonia. J Clin Sleep Med 2013;9:1039-1048.

44 Ferri R, Rundo F, Manconi M, Plazzi G, Brubni O, Oldani A, Ferini-Strambi L, Zucconi M: Improved computation of the atonia index in normal controls and patients with REM sleep behaviour disorder. Sleep Med 2010;11:947949.

45 Berry RB, Brooks R, Gamaldo CE, Harding SM, Marcus VL, Vaughn BV; American Academy of Sleep Medicine: The AASM Manual for the Scoring of Sleep and Associated Events. Rules, Terminology and Technical Specifications, Version 2.0. Darien, American Academy of Sleep Medicine, 2012.

46 Pizza F, Fabbri M, Magosso E, Ursino M, Provini F, Ferri R, Montagna P: Slow eye movements distribution during nocturnal sleep. Clin Neurophysiol 2011;122:1556-1561.
Advanced Analysis of Pharmacosleep Data in Humans
Neuropsychobiology 2015;72:178-187 DOI: $10.1159 / 000431097$ 